\title{
Application of LDPC Erasure Correcting Codes in High Mobility Satellite Communication Yao-Wen WANG ${ }^{1 *}$, Dao-Xing GUO ${ }^{1}$ and Xiao-Kai ZHANG ${ }^{1}$ \\ ${ }^{1}$ PLA University of Science and Technology, China \\ "Email: vladimier@foxmail.com
}

\author{
Keywords: High mobility environment, Satellite communication, Erasure \\ channel, LDPC codes.
}

\begin{abstract}
Providing reliable broadband satellite communications in high mobility environments has attracted considerable attentions in the past few years. One of the biggest challenges is caused by the large Doppler frequency shift due to the rapid relative motion between the transmitters and receivers. The shift, which usually changes with time, can lead to the data loss. The present approaches to mitigate the effect of the shift are concentrated in improving the performance of carrier synchronization, which ensures all the data can be received as much as possible. However, these approaches will exactly increase the complexity of hardware implementation and introduce longer time delay in satellite communication systems. In order to tackle the data loss issues and reduce implementation complexity, we propose a new method in this paper from the perspective of channel coding. Low density parity check (LDPC) codes, which have good performance over erasure channel, are adopted to recover lost data package in high mobility environment. Our algorithm is capable of reducing the complexity of carrier synchronization. The data loss is tolerant and can be recovered by the receiver. The complexity of our proposed algorithm is proportional to code length $\mathrm{n}$. Simulation results show that the channel erasure technique based on LDPC codes can recover the lost data completely within the range of the erasure probability threshold.
\end{abstract}

\section{Introduction}

Current satellite communication (SATcom) systems are mainly designed for communication terminals with low or medium mobility, and high mobility significantly degrades the transmission rate and coverage area. The biggest challenge brought by the high mobility is the performance degradation of carrier synchronization. It is well known that the carrier frequency of SATcom is much higher than that in territory communications, which will make the estimation of the carrier frequency offset much more difficult. Here, we have the equation of Doppler shift: $f_{d}=f_{c} \cdot v / c$, where $f_{c}$ represents the center carrier frequency, $v$ is the speed of the mobile terminal, and $c$ is the speed of light. For example, in S-Band satellite communication systems (with center carrier frequency $f_{c}=2.1 \mathrm{GHz}$ ), if a fighter 
travels at a maximum speed of $v=3.5 \mathrm{Ma}$, then the corresponding Doppler shift is about $8400 \mathrm{~Hz}$, and the first order frequency variation can be $700 \mathrm{~Hz} / \mathrm{s}$. Such huge Doppler shift is close to data transmission rate and will significantly degrade the performances of carrier recovery. Provided the PLL technique is applied in high mobility environment to realize synchronization, a wider loop bandwidth is needed[1]. However, the increase of loop bandwidth will also introduce more noise, which in turn degrades the performances. In most cases, the high mobility will also lead to the PLL on the fly during the data transmission period. Meanwhile, due to the line-of-sight(LOS) propagation property of the transmitted signal wave, the sudden acceleration, rapid climb motion or the sharp turn of mobile terminals, such as planes or missiles, will probably cause the communication link disconnected. In order to recover the lost data in such cases, error control technologies have to be applied. Due to the long time delay of satellite communication, it is reasonable to adopt erasure correcting codes which can effectively avoid the retrans mis sion cost.

The LDPC codes can exhibit an extraordinary performance over a wide range of channels. Some classes of LDPC code ensembles can even asymptotically approach the capacity of the binary erasure channel under iterative decoding algorithm. [2][3]The erasure correcting performances of binary LDPC codes in deep space communication have been explored in[4][5]. Compared with maximum likelihood (ML) algorithm[6], iterative decoding can easily meet the implementation complexity requirements of space communications in terms of high decoding speed. Besides, erasure correcting systems based on LDPC codes can provide an efficient and flexible countermeasure to deal with long fade events. Working on hard values, LDPC erasure correcting codes represent a low-complexity alternative to long channel interleaver.[7]

\section{Erasure Channel and Erasure Correcting Codes}

Elias proposed the model of binary erasure channel $(B E C),[8]$ the input variables set is $\{0,1\}$, while the corresponding output variables set is $\{0,1, E\}$, where $E$ represents erasure. Fig 1 shows the model given by Elias. Each codeword symbol is lost with a fixed constant probability $p$ in transit independent of all the other symbols. Elias had also shown that the capacity of the binary channel is $1-p,[8]$ so that a random linear code can be applied to transmit at any rate $R<1-p$ over the binary erasure channel. In most cases, the locations of erasures are usually known for the receiver. 


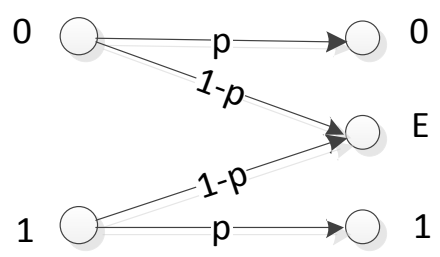

Fig. 1 Binary Erasure Channel

The basic principle of erasure correcting codes is given that, the $k$ source information packages are encoded to $n(n>k)$ packages, if the receiver can accept enough packages, which is a little bigger than $k$, then receiver can recover all the source information packages. Fig 2 gives the general principle of ensemble erasure correcting codes.

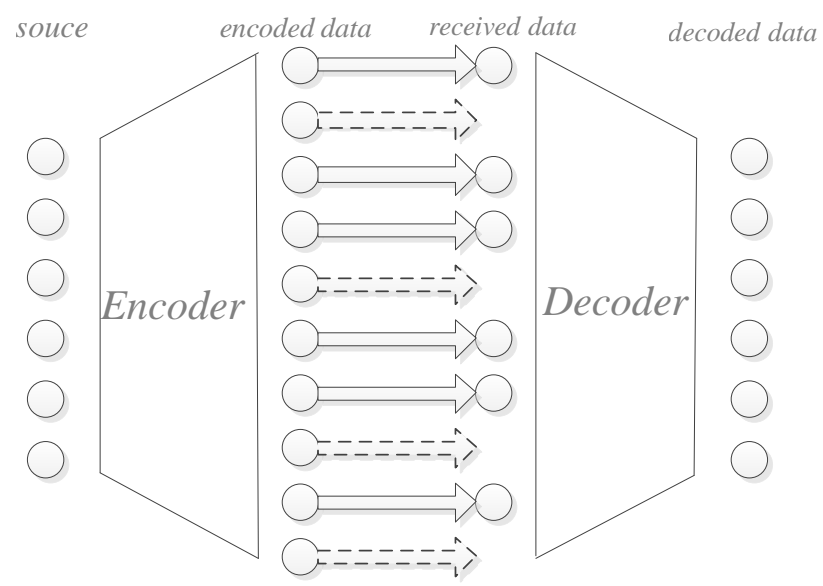

Fig. 2 Princip le of Erasure correcting codes

In this paper, we mainly focus on binary LDPC codes, which exhibit a good performance very close to the capacity over the binary erasure channel.

However, it has to be emphasized that there doesn't exist practical erasure channel at all in real physical channel. It is just a description of channel strategy, which can be thought as a logical channel. The coded data transmitted over the channel in high mobility environment, can be polluted by the noise and Doppler shift. When the Doppler shift varies rapidly, sometimes it will be difficult for the demodulator to tract the frequency offset of the received signal. Meanwhile, if the SNR is too low, the receiver can not work. In such cases, the receiver will then discard the corresponding information bits or packages. That is, the actualperformer of the erasure progress is the receiver, not the practical physical channel.

\section{Algorithm Description}

A LDPC code is a linear block code defined by a sparse parity-check matrix $H$, in which the entries of nonzero elements are sparse. A codeword $x$ has to satisfy the parity-check equation: $H x^{T}=0^{T}$.The Tanner graph representation of the code and its 
associated BP decoding algorithm are presented in[9] and [10]. As suggested by Tanner[11], the LDPC codes can be well illustrated by bipartite graphs. There are two sets of nodes in the graph, one is the set of variable nodes, corresponding to elements of codeword, the other set is check nodes, corresponding to the set of parity-check nodes constraints associated with the codeword. Those for which have the same degree distribution are defined as regular LDPC codes. The bipartite graph determining a $(2,3)$ regular code of length 8 is shown in Fig 3.

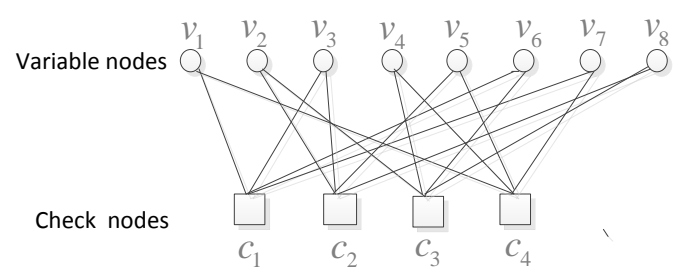

Fig. 3A $(2,3)$ regular LDPC code of length 8 and rate $1 / 2$.

In this paper, the Belief Propagation (BP) decoding algorithm is applied to recover the erasures. LDPC codes are generally desirable, because the belief propagation(BP) decoder is extremely simple and efficient in correcting errors. During the process of decoding, the message is passed between the variable nodes and check nodes. In high mobility environments, we assume that the all the erasures occur in burst and the length of erasured bits is L. The decoder use the received bits, based on the constraining relationship among the received data, to recover the erasured bits. We only think about the hard decision in erasure channel. The decoding algorithm can be descripted as follows:

Step 1.Initializing: All the variable nodes are assigned to 0,1 or $\mathrm{E}$, according to the received value, while all the check variables are assigned to 0 .

Step 2.Direct recovery: To each variable node $v_{i}$, if not erasured, then the value of $v_{i}$ module 2 add to all the check nodes linked, and remove the variable node $v_{i}$ and all the edge connected to it in the bipartite graph. For example, if there is a parity check equation that, $v_{1}+v_{2}+v_{3}=0$, if $v_{3}$ is erasured while $v_{1}$ and $v_{2}$ not, then we will have $v_{3}=v_{1} \oplus v_{2}$.

Step3.Iterative recovery: If the residual bipartite graph exists a degree one parity check node $c_{i}$, then the value of the variable node which connected to $c_{i}$ is declared to be known that $v_{i}=c_{i}$, so an erasured variable node is recovered. Then remove the variable node and the associated edge. Repeat the alternative recovery operations until all the variable nodes are recovered or there exists no parity nodes whose degree is 1 . 
The biggest advantage of iterative decoding algorithm is that it can be encoded and decoded in linear time proportional to the information length n. It only needs XOR operations during the encoding and decoding process, so it can be completed in a linear time. If applied in satellite communications, it can significantly decrease the processing delay of the system.

\section{Simulation Results}

Due to the fluctuation of Doppler shift, it is reasonable to adopt short-length LDPC codes to make sure that the carrier synchronization can maintain during a frame data transmission. Once the synchronization maintaining fails, there will generate erasures and the receiver need to recover the lost data on use of already received data. The LDPC codes selected in this paper is a regular $(1920,960)$ code, which all variable nodes have degree 3 and check nodes have degree 6 . The number of iterations is 30 , and the simulation result is shown below in Fig 4.

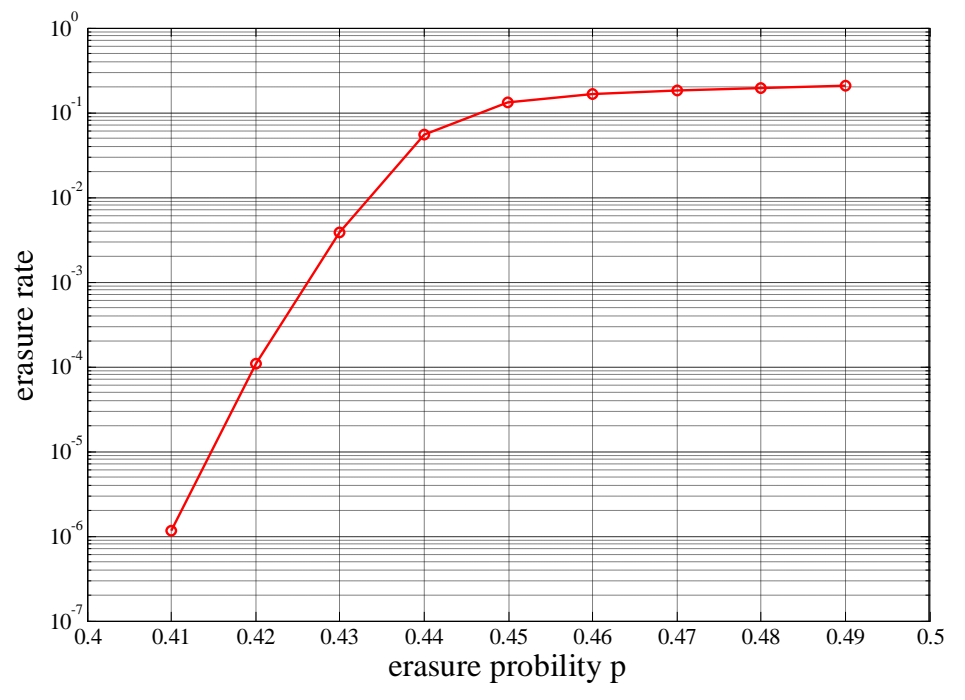

Fig.4 erasure correcting performance of length 1920, rate 1/2 regular LDPC code

It is shown in the figure that, the LDPC code has a good performance over the binary erasure channel. There exists an erasure probability threshold and erasure floor. If the erasure probability $p$ is beyond the threshold, the LDPC codes can recover all the erasured variable nodes. However, if $p$ exceeds the threshold, there will still exist erasured nodes unrecovered even when the number of iterations is increased. Because if the erasure probability exceeded, then there will be too many priori variable nodes are erasured and can not participate in the XOR operation. It can also infer from the figure that, the erasure probability threshold is nearly about 0.41.Besides, the number of iterations is small when the erasure probability is low. 


\section{Conclusion}

In this paper, we have introduced the basic concepts of high mobility satellite communications and pointed out that the biggest challenge is caused by the Doppler shift. There will be inevitable data loss in high mobility environment while the Doppler shift fluctuates rapidly and frequently and the SNR is rather low. Then we propose a feasible solution to resolve the data packet loss. The simulation result shows that the application of LDPC erasure correcting codes can be efficient to recover the data loss in high mobility environment.

\section{References}

[1] Li W, Liu S, Zhou C, et al, High dynamic carrier tracking using Kalman filter aided phase-lock loop[C], IEEE International Conference on Wireless Communications Networking and Mobile Computing(Wicom),2007.

[2] Luby M, MitzenmacherM, Shokrollahi M, Spielman D. Efficient erasure correcting codes. IEEE Transactions on Information Theory 2001;47(2):569-584.

[3] Oswald P, Shokrollahi MA. Capacity-achieving sequences for the erasure channel. IEEE Transations on Information Theory 2002;48(12):364-373.

[4] Henniger.H. Transmission performance analysis of free-spaceoptical communications using gilbert-erasure channel, IEEE.Trans. Comms, 2012; 60 (1): $55-61$.

[5] de Cola T, Paolini E,Liva G, CalzolariG. Reliability options for datacommunications in the future deep-space missions, Proc IEEE 2011; 99(11): 2056 -2074 .

[6] BurshteinD, Miller G. An efficient maximum likelihood decoding of LDPC codes over the binary erasure channel. IEEE Transactions on Information Theory 2004; 50(11):2837-2844.

[7] Alamanac A B, Burzigotti P, De Gaudenzi R, Liva G, Pham. H N, Scalise S. In-deepth analysis of the satellite component of DVB-SH: scenarios, system dimensioning, simulations and field trials results, International J Satellite Commun and Networks 2009;27(4-5):215-240

[8] P.Elias, Coding for two noisy channels, in Information Theory, 3rd London Symp., 1955,pp. 61-76.

[9] R.G.Gallager, Low-density parity-check codes,IRE Trans. Inform. Theory, vol.IT-8, no. 1,pp.21-28, Jan.1962

[10] T.Richardson and R. Urbanke, The capacity of low-density parity-check codes under message-passing decoding, IEEE Trans. Inform. Theory, vol.47,pp.599-618,Feb.2001

[11]M.Tanner,A recursive approach to low complexity codes, IEEE Trans.Inform. Theory, vol.IT-27,pp.533-547,Sept.1981 